\title{
Larger-Scale Transformers for Multilingual Masked Language Modeling
}

\author{
Naman Goyal Jingfei Du Myle Ott Giri Anantharaman Alexis Conneau \\ \{naman, jingfeidu, myleott, giriman, aconneau $@ @$ fb.com
}

\author{
Facebook AI
}

\begin{abstract}
Recent work has demonstrated the effectiveness of cross-lingual language model pretraining for cross-lingual understanding. In this study, we present the results of two larger multilingual masked language models, with 3.5B and 10.7B parameters. Our two new models dubbed XLM-R ${ }_{X L}$ and XLM-R $\mathrm{XXL}_{\mathrm{X}}$ outperform XLM-R by $1.8 \%$ and $2.4 \%$ average accuracy on XNLI. Our model also outperforms the RoBERTa-Large model on several English tasks of the GLUE benchmark by $0.3 \%$ on average while handling 99 more languages. This suggests larger capacity models for language understanding may obtain strong performance on both high- and low-resource languages. We make our code and models publicly available. ${ }^{1}$
\end{abstract}

\section{Introduction}

The goal of this paper is to present a study of the impact of larger capacity models on crosslingual language understanding (XLU). We scale the capacity of XLM-R by almost two orders of magnitude while training on the same $\mathrm{CC} 100$ dataset (Wenzek et al., 2019). Our two new multilingual masked language model dubbed XLM-R $\mathrm{XL}_{\mathrm{XL}}$ and XLM- $\mathrm{RXX}_{\mathrm{XX}}$, with 3.5 and 10.7 billion parameters respectively, significantly outperform the previous XLM-R model on cross-lingual understanding benchmarks and obtain competitive performance with the multilingual T5 models (Raffel et al., 2019; Xue et al., 2020). We show that they can even outperform RoBERTa-Large (Liu et al., 2019) on the GLUE benchmark (Wang et al., 2018).

Recent multilingual masked language models (MLM) like mBERT (Devlin et al., 2018) or XLM (Lample and Conneau, 2019) improved crosslingual language understanding by pretraining large Transformer models (Vaswani et al., 2017) on mul-

\footnotetext{
${ }^{1}$ https://github.com/anonymous
}

tiple languages at once. The XLM-R model (Conneau et al., 2019) extended that approach by scaling the amount of data by two orders of magnitude, from Wikipedia to Common-Crawl and training longer, similar to RoBERTa (Liu et al., 2019). These models are particularly effective for lowresource languages, where both labeled and unlabeled data is scarce. They enable supervised cross-lingual transfer, where labeled data in one language can be used to solve the same task in other languages, and unsupervised cross-lingual transfer, where low-resource language self-supervised representations are improved using additional unlabeled data from higher-resource languages. Furthermore, they reduce the need for training one model per language, and allows the use of a single - potentially much larger - pretrained model that is then fine-tuned on annotated data from many languages.

The better performance of self-supervised crosslingual models on low-resource languages comes however at the cost of lower performance on higherresource languages (Arivazhagan et al., 2019). When the number of languages becomes large, Conneau et al. (2019) even observed an overall decrease of performance on all languages. It was hypothesized that when multilingual models get more capacity, they may showcase strong performance on both high-resource languages and lowresource languages. With only 550M parameters, the XLM-R model is now relatively small compared to new standards. Recent work scaled language models to hundreds of billions (Brown et al., 2020) or even multiple trillion parameters (Fedus et al., 2021), showing consistent gains in doing so. Recently, multilingual T5 showed impressive increase in performance by scaling the model capacity to tens of billions of parameters. Our study complements these findings by showing the impact of larger capacity models on the important pretraining task of multilingual masked language model- 
ing. We show promising results for cross-lingual understanding: XLM-R $\mathrm{XXL}$ can both obtain a new state of the art on some cross-lingual understanding benchmarks and outperform the RoBERTa-Large model on the English GLUE benchmark (Wang et al., 2018). This suggests that very large-scale multilingual models may be able to benefit from the best of both worlds: obtaining strong performance on high-resource languages while still allowing for zero-shot transfer and low-resource language understanding. We make the following contributions:

- We scale XLM capacity by two orders of magnitude, and publicly release XLM- $\mathrm{R}_{\mathrm{XL}}$ and

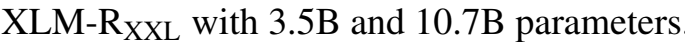

- We show that those two models obtain very strong performance on cross-lingual benchmarks while outperforming RoBERTa Large on the GLUE benchmark.

\section{Pretraining and evaluation}

In this section, we describe the model we use and how we scale it, as well as the data and tasks we use for pretraining and evaluation.

\subsection{Multilingual masked language models}

We use a Transformer model (Vaswani et al., 2017) trained with the multilingual MLM objective (Devlin et al., 2018; Lample and Conneau, 2019) using only monolingual data. We sample streams of text from each language and train the model to predict the masked tokens in the input. We use the same learning procedure as XLM-R. We apply subword tokenization directly on raw text data using Sentence Piece (Kudo and Richardson, 2018) with a unigram language model (Kudo, 2018) just like in XLM-R. We sample batches from different languages using the same sampling distribution as Conneau et al. (2019), with $\alpha=0.3$, and without language embeddings. We use a large vocabulary size of $250 \mathrm{~K}$ with a full softmax and train two different models: XLM-R $\mathrm{XL}_{\mathrm{X}}(\mathrm{L}=36, \mathrm{H}=2560$, A $=32,3.5 \mathrm{~B}$ params $)$ and XLM-R $\mathrm{XXL}_{\mathrm{XL}}(\mathrm{L}=48, \mathrm{H}$ $=4096, \mathrm{~A}=32,10.7 \mathrm{~B}$ params). We pretrain the models on the $\mathrm{CC} 100$ dataset, which corresponds to $167 \mathrm{~B}$ tokens in 100 languages. We compare our approach to previous results as well as the mT5 baselines, which were pretrained on the larger $\mathrm{mC} 4$ corpus of 6.4T tokens.

\subsection{Evaluation}

We consider three evaluation benchmarks. For cross-lingual understanding, we use cross-lingual natural language inference and question answering, and use the GLUE benchmark to evaluate the English performance.

Cross-lingual Natural Language Inference. The XNLI dataset (Conneau et al., 2018) comes with ground-truth dev and test sets in 15 languages, and a ground-truth English training set. The training set has been machine-translated to the remaining 14 languages, providing synthetic training data for these languages as well. We evaluate our model on cross-lingual transfer from English to other languages. We also consider two machine translation baselines: (i) translate-test: dev and test sets are machine-translated to English and a single English model is used (ii) translate-train-all: the English training set is machine-translated to each language and we fine-tune a multilingual model on all training sets. For translations, we use the original XNLI data for consistency.

Cross-lingual Question Answering. We use the MLQA and XQuad benchmark from Lewis et al. (2019) and Artetxe et al. (2019), which extends the English SQuAD benchmark to more languages. We report the F1 score as well as the exact match (EM) score for cross-lingual transfer from English.

The English GLUE Benchmark. Finally, we evaluate the English performance of our model on the GLUE benchmark (Wang et al., 2018) which gathers multiple classification tasks, such as MNLI (Williams et al., 2017), SST-2 (Socher et al., 2013), or QNLI (Rajpurkar et al., 2018).

\subsection{Training details}

We use model parallelism based on tensor parallel (Shoeybi et al., 2019) for scaling models. XLM$\mathrm{R}_{\mathrm{XL}}$ uses model parallel size of 2 and XLM- $\mathrm{R}_{\mathrm{XXL}}$ used 8. Compared to previous XLM-R models, we reduce the batch size and number of updates significantly to keep the compute of the new models similar (see Table 5). For both models, we use batch size of 2048 and train for 500,000 updates. We use pre-LayerNorm setting for both the models which was more stable during training.

For all the tasks in finetuning, we use batch size of 32 and train for 10 epochs. We do early stopping based on the average valid metrics across all languages and report test results. 


\begin{tabular}{|c|c|c|c|c|c|c|c|c|c|c|c|c|c|c|c|c|c|}
\hline Model & Data (\#tok) & en & fr & es & de & el & bg & ru & tr & ar & vi & th & zh & hi & sw & ur & Avg \\
\hline \multicolumn{18}{|c|}{ Fine-tune multilingual model on English training set (Cross-lingual Transfer) } \\
\hline \multirow{2}{*}{$\begin{array}{l}\text { mBERT } \\
\text { XLM }\end{array}$} & \multirow{2}{*}{ Wikipedia } & 80.8 & 64.3 & 68.0 & 70.0 & 65.3 & 73.5 & 73.4 & 58.9 & 67.8 & 49.7 & 54.1 & 60.9 & 57.2 & 69.3 & 67.8 & 65.4 \\
\hline & & 83.2 & 76.5 & 76.3 & 74.2 & 73.1 & 74.0 & 73.1 & 67.8 & 68.5 & 71.2 & 69.2 & 71.9 & 65.7 & 64.6 & 63.4 & 71.5 \\
\hline mT5-Base & \multirow{4}{*}{$\begin{array}{c}\mathrm{mC} 4 \\
(6.4 \mathrm{~T})\end{array}$} & 84.7 & 73.3 & 78.6 & 77.4 & 77.1 & 80.3 & 79.1 & 70.8 & 77.1 & 69.4 & 73.2 & 72.8 & 68.3 & 74.2 & 74.1 & 75.4 \\
\hline mT5-Large & & 89.4 & 79.8 & 84.1 & 83.4 & 83.2 & 84.2 & 84.1 & 77.6 & 81.5 & 75.4 & 79.4 & 80.1 & 73.5 & 81.0 & 80.3 & 81.1 \\
\hline mT5-XL & & 90.6 & 82.2 & 85.4 & 85.8 & 85.4 & 81.3 & 85.3 & 80.4 & 83.7 & 78.6 & 80.9 & 82.0 & 77.0 & 81.8 & 82.7 & 82.9 \\
\hline mT5-XXL & & 91.6 & 84.5 & 87.7 & 87.3 & 87.3 & 87.8 & 86.9 & 83.2 & 85.1 & 80.3 & 81.7 & 83.8 & 79.8 & 84.6 & 83.6 & 84.5 \\
\hline XLM-R Base & \multirow{4}{*}{$\begin{array}{l}\text { CC100 } \\
(167 B)\end{array}$} & 85.8 & 79.7 & 80.7 & 78.7 & 77.5 & 79.6 & 78.1 & 74.2 & 73.8 & 76.5 & 74.6 & 76.7 & 72.4 & 66.5 & 68.3 & 76.2 \\
\hline XLM-R & & 89.1 & 84.1 & 85.1 & 83.9 & 82.9 & 84.0 & 81.2 & 79.6 & 79.8 & 80.8 & 78.1 & 80.2 & 76.9 & 73.9 & 73.8 & 80.9 \\
\hline XLM-R ${ }_{X L}$ & & 90.7 & 85.5 & 86.5 & 84.6 & 84.0 & 85.2 & 82.7 & 81.7 & 81.6 & 82.4 & 79.4 & 81.7 & 78.5 & 75.3 & 74.3 & 82.3 \\
\hline XLM-R XXL & & 91.6 & 86.2 & 87.3 & 87.0 & 85.1 & 85.7 & 82.5 & 82.0 & 82.5 & 83.0 & 79.5 & 82.6 & 79.8 & 76.2 & 74.9 & 83.1 \\
\hline \multicolumn{18}{|c|}{ Translate everything to English and use English-only model (TRANSLATE-TEST) } \\
\hline RoBERTa & & 91.3 & 82.9 & 84.3 & 81.2 & 81.7 & 83.1 & 78.3 & 76.8 & 76.6 & 74.2 & 74.1 & 77.5 & 70.9 & 66.7 & 66.8 & 77.8 \\
\hline \multicolumn{18}{|c|}{ Fine-tune multilingual model on all training sets (TRANSLATE-TRAIN-ALL) } \\
\hline & \multirow{4}{*}{$\begin{array}{c}\mathrm{mC} 4 \\
(6.4 \mathrm{~T})\end{array}$} & 82.0 & 74.4 & 78.5 & 77.7 & 78.1 & 79.1 & 77.9 & 72.2 & 76.5 & 71.5 & 75.0 & 74.8 & 70.4 & 74.5 & 76.0 & 75.9 \\
\hline mT5-Large & & 88.3 & 80.3 & 84.1 & 84.0 & 83.7 & 84.9 & 83.8 & 79.8 & 82.0 & 76.4 & 79.9 & 81.0 & 75.9 & 81.3 & 81.7 & 81.8 \\
\hline mT5-XL & & 90.9 & 84.2 & 86.8 & 86.8 & 86.4 & 87.4 & 86.8 & 83.1 & 84.9 & 81.3 & 82.3 & 84.4 & 79.4 & 83.9 & 84.0 & 84.8 \\
\hline mT5-XXL & & 92.7 & 87.2 & 89.4 & 89.8 & 89.5 & 90.0 & 89.1 & 86.5 & 87.6 & 84.3 & 85.6 & 87.1 & 83.8 & 87.5 & 86.5 & 87.8 \\
\hline XLM-R Base & \multirow{4}{*}{$\begin{array}{l}\text { CC100 } \\
(167 B)\end{array}$} & 85.4 & 81.4 & 82.2 & 80.3 & 80.4 & 81.3 & 79.7 & 78.6 & 77.3 & 79.7 & 77.9 & 80.2 & 76.1 & 73.1 & 73.0 & 79.1 \\
\hline XLM-R Large & & 89.1 & 85.1 & 86.6 & 85.7 & 85.3 & 85.9 & 83.5 & 83.2 & 83.1 & 83.7 & 81.5 & 83.7 & 81.6 & 78.0 & 78.1 & 83.6 \\
\hline XLM-R ${ }_{\mathrm{XL}}$ & & 91.1 & 87.2 & 88.1 & 87.0 & 87.4 & 87.8 & 85.3 & 85.2 & 85.3 & 86.2 & 83.8 & 85.3 & 83.1 & 79.8 & 78.2 & 85.4 \\
\hline XLM-R XXL & & 91.5 & 87.6 & 88.7 & 87.8 & 87.4 & 88.2 & 85.6 & 85.1 & 85.8 & 86.3 & 83.9 & 85.6 & 84.6 & 81.7 & 80.6 & 86.0 \\
\hline
\end{tabular}

Table 1: Results on cross-lingual classification (XNLI). We report the accuracy on each of the 15 XNLI languages and average accuracy, and specify the dataset and its corresponding size in number of tokens. We report results of XLM-R models with increasing capacity, from 270M (Base), 550M (Large), 3.5B (XL) to 10.7B (XXL) parameters.

\section{Analysis and Results}

In this section, we present our results and compare XLM-R $\mathrm{XL}_{\mathrm{L}}$ and XLM-R $\mathrm{XXL}$ performance to other methods from previous work.

Cross-lingual understanding results. On XNLI, we observe in Table 1 that scaling the capacity from XLM-R $\mathrm{R}_{\text {Large }}$ to XLM-R $\mathrm{XL}_{\mathrm{XL}}$ leads to an average accuracy improvement of 1.4 on zero-shot cross-lingual transfer and 1.8 on multilingual fine-tuning. When scaling even further to XLM-R $\mathrm{XXL}_{\mathrm{X}}$, we observe a total improvement of 2.2 on zero-shot and 2.4 on translate-train-all compared to XLM-R $\mathrm{XL}_{\mathrm{L}}$, with a new state of the art on French, Vietnamese and Hindi. On MLQA, in Table 4, we observe even larger gains for cross-lingual zero-shot transfer, where scaling from XLM- $\mathrm{R}_{\text {Large }}$ to XLM-R $\mathrm{XXL}_{\mathrm{X}}$ leads to improvements of $4.1 \mathrm{~F} 1$ and $3.9 \mathrm{EM}$ scores on average. Similarly, on XQuad we observe improvements of $4.4 \mathrm{~F} 1$ and 5.5 scores, with new state-of-the-art results on Arabic, German, Greek and Russian (see Table 3).

Comparison to monolingual English model. For smaller-capacity models like the Base and Large version of XLM-R, it was shown that the more languages are considered the lower the perfor- mance (Conneau et al., 2019), in particular on highresource languages. For instance, XLM-R $\mathrm{R}_{\text {Large }}$ was outperformed by RoBERTa $a_{\text {Large }}$ by $1 \%$ accuracy on average on several downstream tasks from the GLUE benchmark, as illustrated in Table2. With larger capacity, we now observe that XLM- $\mathrm{R}_{\mathrm{XXL}}$ is able to outperform RoBERTa Large by $0.3 \mathrm{dev}$ points, going from 92.9 to 93.2 average accuracy, while handling 99 more languages. While a RoBERTaxxL model may outperform XLM-R $\mathrm{XXL}_{\mathrm{X}}$, we believe it interesting to notice that with more capacity, a multilingual model can get strong high-resource performance while not losing its cross-lingual transfer ability for lower-resource languages. Given the compute needed for training such large-scale models, the possibility of training a single very large model on hundreds of languages with state-of-the-art performance on high-resource languages is an encouraging and positive result.

\begin{tabular}{l|c|ccccc|c}
\hline Model & \#lgs & MNLI & QNLI & QQP & SST & MRPC & Avg \\
\hline RoBERTa $^{\dagger}$ & 1 & 90.2 & 94.7 & 92.2 & 96.4 & $\mathbf{9 0 . 9}$ & 92.9 \\
XLM-R $_{\text {Large }}$ & 100 & 88.9 & 93.8 & 92.3 & 95.0 & 89.5 & 91.9 \\
XLM-R $_{X L}$ & 100 & 90.4 & 94.9 & 92.5 & 96.6 & 90.4 & 93.0 \\
XLM-R $_{X X L}$ & 100 & $\mathbf{9 0 . 9}$ & $\mathbf{9 5 . 0}$ & $\mathbf{9 2 . 6}$ & $\mathbf{9 6 . 7}$ & 90.7 & $\mathbf{9 3 . 2}$ \\
\hline
\end{tabular}

Table 2: GLUE dev results 


\begin{tabular}{|c|c|c|c|c|c|c|c|c|c|c|c|c|}
\hline Model & en & ar & de & el & es & hi & ru & th & tr & vi & zh & avg \\
\hline \multicolumn{13}{|c|}{ Cross-lingual zero-shot transfer (models fine-tune on English data only) } \\
\hline mT5-Large & $88.4 / 77.3$ & $75.2 / 56.7$ & $80.0 / 62.9$ & $77.5 / 57.6$ & $81.8 / 64.2$ & 73.4 / 56.6 & $74.7 / 56.9$ & $73.4 / 62.0$ & $76.5 / 56.3$ & 79.4 / 60.3 & $75.9 / 65.5$ & $77.8 / 61.5$ \\
\hline mT5-XL & $88.8 / 78.1$ & 77.4 / 60.8 & $80.4 / 63.5$ & $80.4 / 61.2$ & $82.7 / 64.5$ & $76.1 / 60.3$ & $76.2 / 58.8$ & $74.2 / 62.5$ & $77.7 / 58.4$ & $80.5 / 60.8$ & $80.5 / 71.0$ & 79.5 / 63.6 \\
\hline $\mathrm{mt} 5-\mathrm{XXL}$ & $90.9 / 80.1$ & $80.3 / 62.6$ & $\mathbf{8 3 . 1} / 65.5$ & $83.3 / 65.5$ & $85.1 / 68.1$ & $81.7 / 65.9$ & $79.3 / 63.6$ & $77.8 / 66.1$ & $80.2 / 60.9$ & $83.1 / 63.6$ & $83.1 / 73.4$ & $82.5 / 66.8$ \\
\hline XLM-R Large & $86.5 / 75.7$ & $68.6 / 49.0$ & 80.4 / 63.4 & $79.8 / 61.7$ & $82.0 / 63.9$ & $76.7 / 59.7$ & $80.1 / 64.3$ & $74.2 / 62.8$ & $75.9 / 59.3$ & $79.1 / 59.0$ & $59.3 / 50.0$ & $76.6 / 60.8$ \\
\hline XLM-RXL & $89.5 / 79.0$ & 78.4 / 61.6 & $81.3 / 64.1$ & $82.3 / 63.9$ & $84.6 / 66.2$ & $78.8 / 63.2$ & $81.5 / 65.0$ & $76.0 / 65.5$ & $73.9 / 57.9$ & $81.7 / 61.8$ & $72.3 / 66.1$ & $80.0 / 64.9$ \\
\hline XLM-R ${ }_{X X L}$ & $89.3 / 79.4$ & $80.1 / \mathbf{6 3 . 7}$ & 82.7 / 65.8 & $83.4 / 65.5$ & $83.8 / 66.0$ & $80.7 / 65.4$ & $82.4 / 65.4$ & $76.6 / 65.6$ & $76.8 / \mathbf{6 1 . 7}$ & $82.2 / 63.0$ & $74.1 / 67.4$ & $81.1 / 66.3$ \\
\hline
\end{tabular}

Table 3: XQuad results (F1/EM) for each language.

\begin{tabular}{|c|c|c|c|c|c|c|c|c|}
\hline Model & en & es & de & ar & hi & vi & zh & Avg \\
\hline \multicolumn{9}{|c|}{ Cross-lingual zero-shot transfer (models fine-tune on English data only) } \\
\hline mT5-Large & $84.9 / 70.7$ & $65.3 / 44.6$ & $68.9 / 51.8$ & $73.5 / 54.1$ & $66.9 / 47.7$ & $72.5 / 50.7$ & $66.2 / 42.0$ & $71.2 / 51.7$ \\
\hline mT5-XL & $85.5 / 71.9$ & $68.0 / 47.4$ & $70.5 / 54.4$ & $75.2 / 56.3$ & $70.5 / 51.0$ & $74.2 / 52.8$ & $70.5 / 47.2$ & $73.5 / 54.4$ \\
\hline mT5-XXL & $86.7 / 73.5$ & $70.7 / 50.4$ & $74.0 / 57.8$ & $76.8 / 58.4$ & $75.6 / 57.3$ & $76.4 / 56.0$ & $71.8 / 48.8$ & $76.0 / 57.4$ \\
\hline XLM-R Large & $80.6 / 67.8$ & $74.1 / 56.0$ & $68.5 / 53.6$ & $63.1 / 43.5$ & $69.2 / 51.6$ & $71.3 / 50.9$ & $68.0 / 45.4$ & $70.7 / 52.7$ \\
\hline$X L M-R_{X L}$ & $85.1 / 72.6$ & $66.7 / 46.2$ & $70.5 / 55.5$ & $74.3 / 56.9$ & $72.2 / 54.7$ & $74.4 / 52.9$ & $70.9 / 48.5$ & $73.4 / 55.3$ \\
\hline$X L M-R_{X X L}$ & $85.5 / 72.4$ & $68.6 / 48.4$ & $72.7 / 57.8$ & $75.4 / 57.6$ & $73.7 / 55.8$ & $76.0 / 55.0$ & $71.7 / 48.9$ & $74.8 / 56.6$ \\
\hline
\end{tabular}

Table 4: MLQA results (F1/EM) for each language.

Discussion and comparison to mT5. Both mT5 and XLM-R models obtain strong performance on cross-lingual understanding benchmarks, as well as high performance on English benchmarks (see the score of 91.6 of $\mathrm{mT} 5 \mathrm{XXL}$ on English XNLI). Many hyperparameters are however different between mT5 and XLM-R models which makes difficult an apple-to-apple comparison. First, as shown in Table 5, the mT5 models are pretrained on the much larger mC4 dataset which contains around 6.4T tokens, which is 38 times bigger than $\mathrm{CC} 100$ (167B tokens). While XLM- $\mathrm{R}_{\text {Large }}$ was pretrained with more updates (6T tokens), the XLM-R $\mathrm{XL}_{\mathrm{XL}}$ and XLM-R XXL models have seen less tokens (0.5T) during pretraining than their mT5 counterparts, although it also uses a bigger batch size (2048 over 1024 for mT5). Another difference is the context sequence length of 512 for XLM-R and 1024 for mT5. The mT5-XXL model also has slightly more parameters (13B over 10.7B). The larger number of updates combined with the larger dataset size may explain the larger improvement from the XL model to the XXL model in the case of mT5 $(+3 \mathrm{av}-$ erage accuracy on XNLI), in which the additional

\begin{tabular}{l|c|c|c|c|c|c}
\hline Model & $\begin{array}{c}\text { Number of } \\
\text { parameters }\end{array}$ & $\begin{array}{c}\text { Dataset } \\
\text { name }\end{array}$ & $\begin{array}{c}\text { Dataset } \\
\text { size }\end{array}$ & $\begin{array}{c}\text { Number of } \\
\text { training tokens }\end{array}$ & $\begin{array}{c}\text { Batch } \\
\text { size }\end{array}$ & $\begin{array}{c}\text { Sequence } \\
\text { length }\end{array}$ \\
\hline XLM- $\mathrm{R}_{\text {Large }}$ & $550 \mathrm{M}$ & $\mathrm{CC} 100$ & $167 \mathrm{~B}$ & $6 \mathrm{~T}$ & 8192 & 512 \\
XLM-R & $3.5 \mathrm{~B}$ & $\mathrm{CC} 100$ & $167 \mathrm{~B}$ & $0.5 \mathrm{~T}$ & 2048 & 512 \\
XLM-R & $10.7 \mathrm{~B}$ & $\mathrm{CC100}$ & $167 \mathrm{~B}$ & $0.5 \mathrm{~T}$ & 2048 & 512 \\
mt5-XL & $3.7 \mathrm{~B}$ & $\mathrm{mC4}$ & $6.4 \mathrm{~T}$ & $1 \mathrm{~T}$ & 1024 & 1024 \\
mt5-XXL & $13 \mathrm{~B}$ & $\mathrm{mC4}$ & $6.4 \mathrm{~T}$ & $1 \mathrm{~T}$ & 1024 & 1024 \\
\hline
\end{tabular}

Table 5: Comparison of datasets and pretraining details between XLM-R and mT5. We report dataset sizes and number of updates in terms of number of tokens. capacity can exploit the large quantity of unlabeled $\mathrm{mC} 4$ data. We note however that the $\mathrm{mT} 5_{\mathrm{XL}}$ is outperformed by XLM-R $\mathrm{XL}$ on XNLI by $0.6 \%$ on average, on XQuad by $1.3 \%$ and on MLQA by $0.9 \%$ when considering average EM score. In comparison, gains of XLM-R from the XL to the XXL architecture are only of 0.6 on average. Another explanation may be that generative models scale better than masked language models. The difference in the nature of the pretraining dataset is particularly striking when looking at the variance of performance across languages. For example the mT5XXL outperforms XLM-R XXL $_{\text {by }} 8.4$ points on Swahili on XNLI zero-shot, while it only outperforms XLM-R XXL by 1.4 average accuracy. These results may suggest that the $\mathrm{CC} 100$ dataset gets saturated with current larger-capacity models.

\section{Conclusion}

In this study, we scaled the model capacity of the XLM-R model up to 10.7B parameters and obtained stronger performance than previous XLM$\mathrm{R}$ models on cross-lingual understanding benchmarks. We also show that the additional capacity allows a multilingual model to outperform a the RoBERTa Large baseline on English benchmarks. Our technical study thus suggests that larger capacity multilingual model can obtain state-of-the-art cross-lingual understanding results while maintaining strong performance on high-resource languages. Our work provides an alternative to mT5 models, with new state-of-the-art performance on some languages. We release our code and models publicly. 


\section{References}

Naveen Arivazhagan, Ankur Bapna, Orhan Firat, Dmitry Lepikhin, Melvin Johnson, Maxim Krikun, Mia Xu Chen, Yuan Cao, George Foster, Colin Cherry, et al. 2019. Massively multilingual neural machine translation in the wild: Findings and challenges. arXiv preprint arXiv:1907.05019.

Mikel Artetxe, Sebastian Ruder, and Dani Yogatama. 2019. On the cross-lingual transferability of monolingual representations. arXiv preprint arXiv: 1910.11856.

Tom B Brown, Benjamin Mann, Nick Ryder, Melanie Subbiah, Jared Kaplan, Prafulla Dhariwal, Arvind Neelakantan, Pranav Shyam, Girish Sastry, Amanda Askell, et al. 2020. Language models are few-shot learners. Proc. of NeurIPS.

Alexis Conneau, Kartikay Khandelwal, Naman Goyal, Vishrav Chaudhary, Guillaume Wenzek, Francisco Guzmán, Edouard Grave, Myle Ott, Luke Zettlemoyer, and Veselin Stoyanov. 2019. Unsupervised cross-lingual representation learning at scale. arXiv preprint arXiv:1911.02116.

Alexis Conneau, Ruty Rinott, Guillaume Lample, Adina Williams, Samuel R. Bowman, Holger Schwenk, and Veselin Stoyanov. 2018. Xnli: Evaluating crosslingual sentence representations. In EMNLP. Association for Computational Linguistics.

Jacob Devlin, Ming-Wei Chang, Kenton Lee, and Kristina Toutanova. 2018. Bert: Pre-training of deep bidirectional transformers for language understanding. NAACL.

William Fedus, Barret Zoph, and Noam Shazeer. 2021. Switch transformers: Scaling to trillion parameter models with simple and efficient sparsity. arXiv preprint arXiv:2101.03961.

Taku Kudo. 2018. Subword regularization: Improving neural network translation models with multiple subword candidates. In $A C L$, pages 66-75.

Taku Kudo and John Richardson. 2018. Sentencepiece: A simple and language independent subword tokenizer and detokenizer for neural text processing. EMNLP.

Guillaume Lample and Alexis Conneau. 2019. Crosslingual language model pretraining. NeurIPS.

Patrick Lewis, Barlas Oğuz, Ruty Rinott, Sebastian Riedel, and Holger Schwenk. 2019. Mlqa: Evaluating cross-lingual extractive question answering. arXiv preprint arXiv: 1910.07475.

Yinhan Liu, Myle Ott, Naman Goyal, Jingfei Du, Mandar Joshi, Danqi Chen, Omer Levy, Mike Lewis, Luke Zettlemoyer, and Veselin Stoyanov. 2019. Roberta: A robustly optimized BERT pretraining approach. arXiv preprint arXiv:1907.11692.
Colin Raffel, Noam Shazeer, Adam Roberts, Katherine Lee, Sharan Narang, Michael Matena, Yanqi Zhou, Wei Li, and Peter J. Liu. 2019. Exploring the limits of transfer learning with a unified text-to-text transformer. arXiv preprint arXiv:1910.10683.

Pranav Rajpurkar, Robin Jia, and Percy Liang. 2018. Know what you don't know: Unanswerable questions for squad. $A C L$.

Mohammad Shoeybi, Mostofa Patwary, Raul Puri, Patrick LeGresley, Jared Casper, and Bryan Catanzaro. 2019. Megatron-lm: Training multi-billion parameter language models using model parallelism. arXiv preprint arXiv:1909.08053.

Richard Socher, Alex Perelygin, Jean Wu, Jason Chuang, Christopher D Manning, Andrew Ng, and Christopher Potts. 2013. Recursive deep models for semantic compositionality over a sentiment treebank. In EMNLP, pages 1631-1642.

Ashish Vaswani, Noam Shazeer, Niki Parmar, Jakob Uszkoreit, Llion Jones, Aidan N. Gomez, Lukasz Kaiser, and Illia Polosukhin. 2017. Attention is all you need. In Advances in Neural Information Processing Systems, pages 6000-6010.

Alex Wang, Amanpreet Singh, Julian Michael, Felix Hill, Omer Levy, and Samuel R Bowman. 2018. Glue: A multi-task benchmark and analysis platform for natural language understanding. arXiv preprint arXiv:1804.07461.

Guillaume Wenzek, Marie-Anne Lachaux, Alexis Conneau, Vishrav Chaudhary, Francisco Guzman, Armand Joulin, and Edouard Grave. 2019. Ccnet: Extracting high quality monolingual datasets from web crawl data. arXiv preprint arXiv:1911.00359.

Adina Williams, Nikita Nangia, and Samuel R Bowman. 2017. A broad-coverage challenge corpus for sentence understanding through inference. Proceedings of the 2nd Workshop on Evaluating VectorSpace Representations for NLP.

Linting Xue, Noah Constant, Adam Roberts, Mihir Kale, Rami Al-Rfou, Aditya Siddhant, Aditya Barua, and Colin Raffel. 2020. mt5: A massively multilingual pre-trained text-to-text transformer. arXiv preprint arXiv:2010.11934. 